\title{
Endoscopic complete closure of duodenal mucosal defects using a clip with a looped thread after endoscopic resection
}
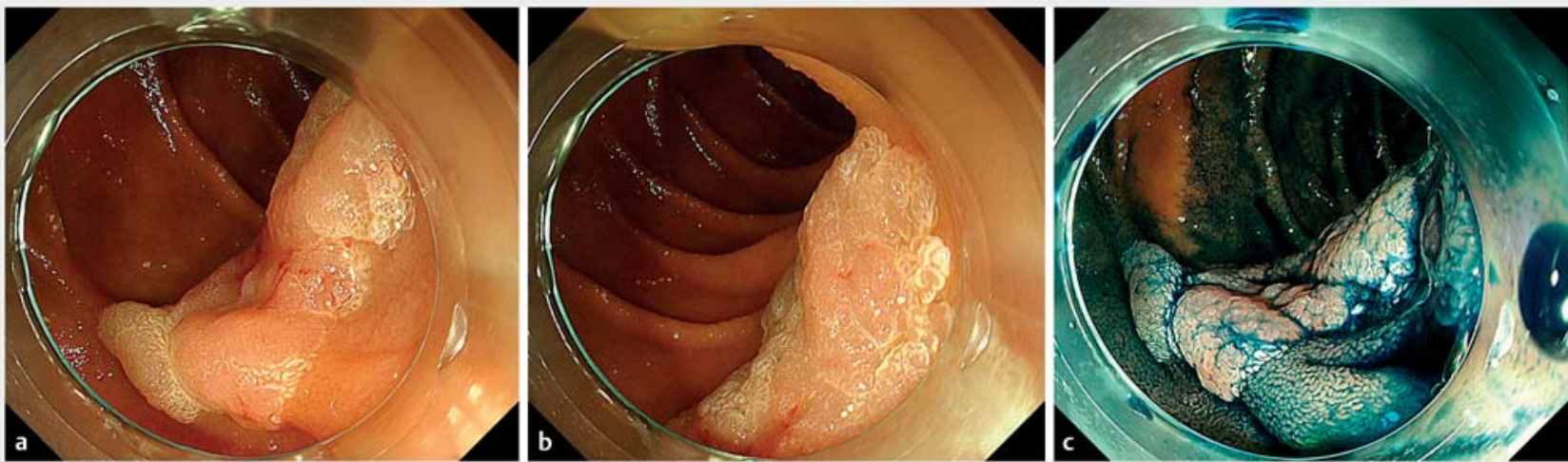

- Fig. 1 Endoscopic images showing a flat elevated lesion in the second portion of the duodenum. a, b In white light. c In chromoendoscopy.

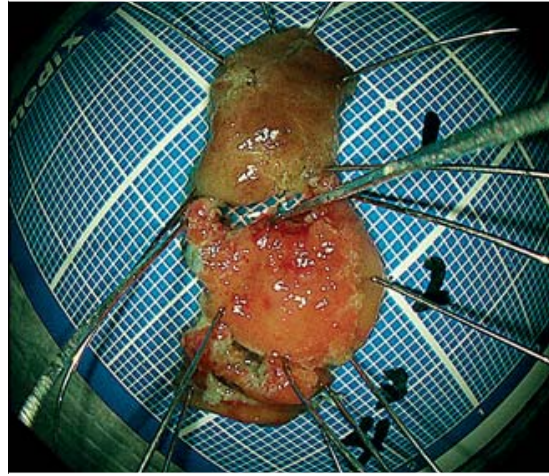

- Fig. 2 The specimen was resected by piecemeal endoscopic mucosal resection.

Closure of mucosal defects after endoscopic resection of superficial nonampullary duodenal tumors is important to reduce the risk of delayed bleeding and perforation [1]. Therefore, we developed a new closure method using a clip with a looped thread ( $\vee$ Video 1 ).

An 84-year-old man had a flat elevated $35-\mathrm{mm}$ lesion in the second portion of the duodenum ( $\mathbf{F i g . 1 a , b , c ) . ~ A ~ b i o p s y ~}$ revealed it was an adenoma; therefore, we performed piecemeal endoscopic mucosal resection using a gastroscope (GIF-H290T; Olympus, Tokyo, Japan) with an endoscopic cap (TOP Endoscopic Hood; TOP, Tokyo, Japan) and bipolar snares (BSDA-217, BSDES-127: diameter 10, $26 \mathrm{~mm}$, resp.; Zeon Corporation,
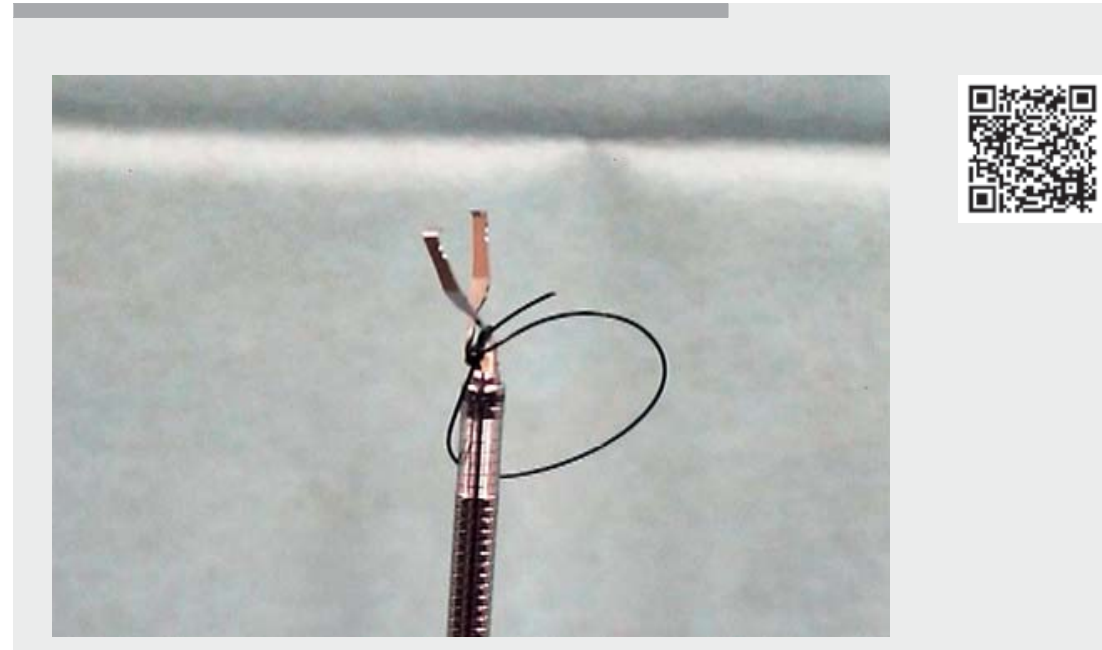

Video 1 Mucosal closure after endoscopic resection of a duodenal tumor using a clip with a looped thread.

Tokyo, Japan) (> Fig.2). There were no adverse events; however, closure of the mucosal defect after endoscopic resection was difficult using only clips because the mucosal defect was large ( $>$ Fig. $\mathbf{3 a}$ ). Therefore, we attempted endoscopic closure as follows. First, a looped nylon suture was tied to a clip (HX-610-090; Olympus) mounted on an applicator (HX-110LR; Olympus). The clip was then retracted into the applicator and inserted into the accessory channel before being placed on the proximal side of the defect ( $\vee$ Fig.3 b). Another clip was used to anchor the suture to the distal normal mucosa ( $\triangleright$ Fig. $\mathbf{3 c}$ ), and additional clips were placed to achieve complete closure ( $\triangleright$ Fig.3d,e). The nylon suture was cut using scissor forceps (FS-3L-1; Olympus), and complete closure was achieved without delayed adverse events.

Various procedures for mucosal closure exist, such as a simple closure technique using clips, clips with a string [2], endoloop [3], or over-the-scope clip [4]. This is a modified method of traction using a 

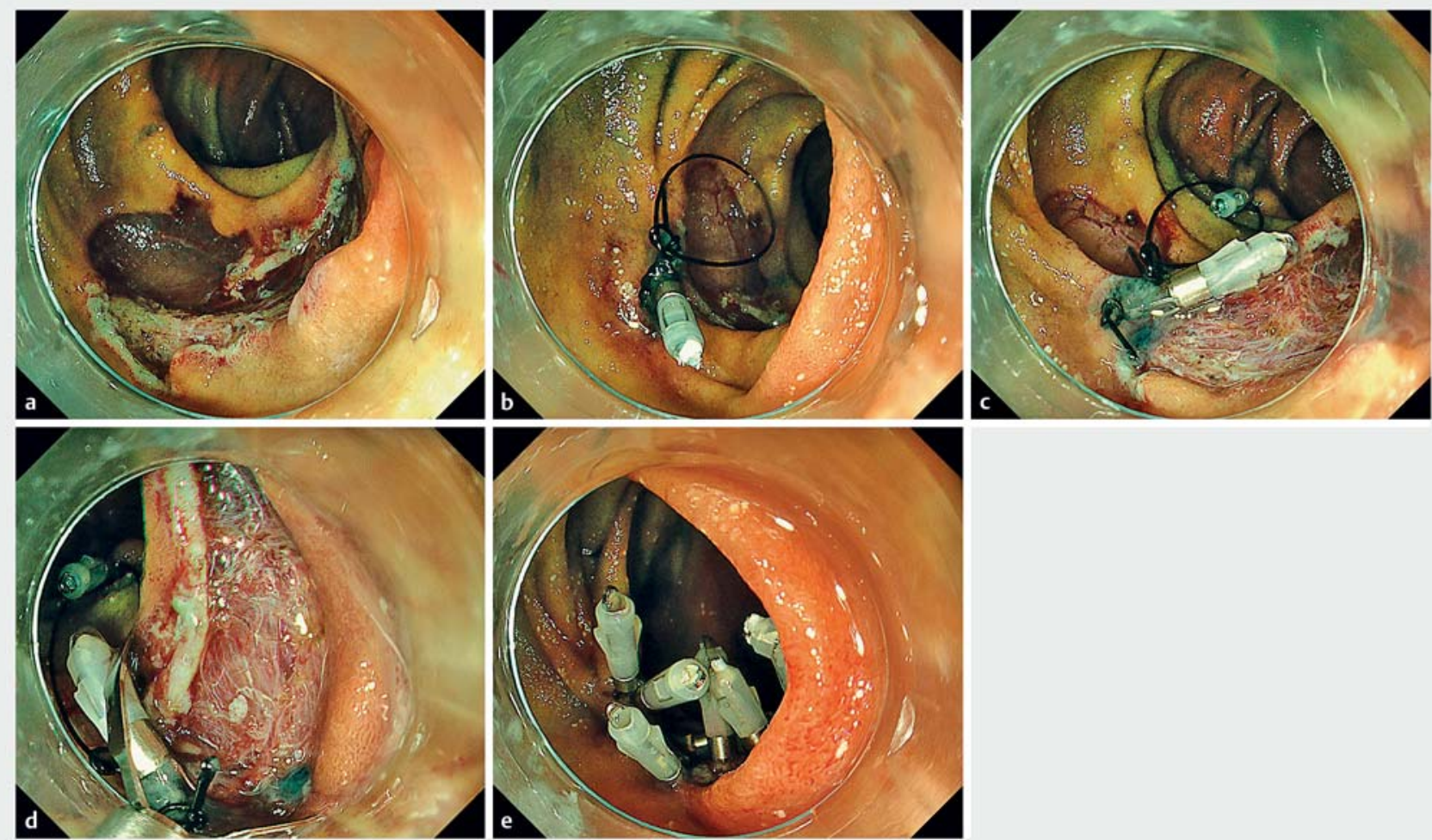

- Fig. 3 How to perform endoscopic closure assisted by a clip with a looped thread. a Large mucosal defect. b The clip with a looped nylon suture is placed on the proximal side of the mucosal defect. $\mathbf{c}$ Another clip is used to anchor the line to the normal mucosa distal to the mucosal defect. $\mathbf{d}$ Additional clips are placed. e Complete closure is achieved.

clip with a looped thread during colorectal endoscopic submucosal dissection [5], and is similar to using clips with a string [2]. In this method the thread does not have to be placed in the accessory channel, so the gastroscope is maneuverable. Furthermore, dual-accessory channel endoscopes and other expensive devices are not required.

\section{Endoscopy_UCTN_Code_TTT_1AO_2AI}

\section{Competing interests}

The authors declare that they have no conflict of interest.

The authors

Kengo Kasuga ${ }^{1}$, Ichiro Oda ${ }^{1}$, Satoru Nonaka ${ }^{1}$, Seiichiro Abe ${ }^{1}$, Haruhisa Suzuki ${ }^{1}$, Toshio Uraoka², Yutaka Saito ${ }^{1}$

1 Endoscopy Division, National Cancer Center Hospital, Tokyo, Japan
2 Department of Gastroenterology and Hepatology, Gunma University Graduate School of Medicine, Maebashi, Japan

\section{Corresponding author}

\section{Ichiro Oda, MD}

Endoscopy Division, National Cancer

Center Hospital, 5-1-1 Tsukiji, Chuo-ku,

Tokyo 104-0045, Japan

Fax: +81335423815

ioda@ncc.go.jp

\section{References}

[1] Tsutsumi K, Kato M, Kakushima N et al. Efficacy of endoscopic preventive procedures to reduce delayed adverse events after endoscopic resection of superficial nonampullary duodenal epithelial tumors: a meta-analysis of observational comparative trials. Gastrointest Endosc 2021; 93: 367-374.e3

[2] Nishizawa T, Akimoto T, Uraoka T et al. Endoscopic string clip suturing method: a prospective pilot study (with video). Gastrointest Endosc 2018; 87: 1074-1078
[3] Ye LP, Mao XL, Zheng HH et al. Safety of endoscopic resection for duodenal subepithelial lesions with wound closure using clips and an endoloop: an analysis of 68 cases. Surg Endosc 2017; 31: 1070-1077

[4] Tashima T, Ohata K, Sakai E et al. Efficacy of an over-the-scope clip for preventing adverse events after duodenal endoscopic submucosal dissection: a prospective interventional study. Endoscopy 2018; 50: 487496

[5] Indo N, Anami T, Asaji N et al. Easy and effective counter-traction using a clip with a looped thread for colorectal endoscopic submucosal dissection. Endoscopy 2019; 51: E233-E234

\section{Bibliography}

Endoscopy 2022; 54: E135-E136

DOI $10.1055 / \mathrm{a}-1443-4585$

ISSN 0013-726X

published online 20.4 .2021

(c) 2021. Thieme. All rights reserved.

Georg Thieme Verlag KG, Rüdigerstraße 14,

70469 Stuttgart, Germany 